\title{
Robot Ontologies for Sensor- and Image-Guided Surgery
}

\author{
Tamás Haidegger*, Marcos Barreto $^{\dagger}$, Paulo J. S. Gonçalves ${ }^{\ddagger}$, Maki K. Habib ${ }^{\aleph}$, \\ S. Veera Ragavan ${ }^{£}$, Howard $\mathrm{Li}^{\S}$, Alberto Vaccarella ", Roberta Perroneף, Edson Prestes ${ }^{\nabla}$
}

\begin{abstract}
Robots and robotics are becoming more complex and flexible, due to technological advancement, improved sensing capabilities and machine intelligence. Service robots target a wide range of applications, relying on advanced Human-Robot Interaction. Medical robotics is becoming a leading application area within, and the number of surgical, rehabilitation and hospital assistance robots is rising rapidly. However, the complexity of the medical environment has been a major barrier, preventing a wider use of robotic technology, thus mostly teleoperated, human-in-the-loop control solutions emerged so far. Providing smarter and better medical robots requires a systematic approach in describing and translating human processes for the robots. It is believed that ontologies can bridge human cognitive understanding and robotic reasoning (machine intelligence). Besides, ontologies serve as a tool and method to assess the added value robotic technology brings into the medical environment. The purpose of this paper is to identify relevant ontology research in medical robotics, and to review the state-of-the art. It focuses on the surgical domain, fundamental terminology and interactions are described for two example applications in neurosurgery and orthopaedics.
\end{abstract}

Keywords: Robot ontologies, cognitive robots, surgical robotics, image-guided surgery.

\section{INTRODUCTION}

Service robots with increased capabilities find numerous applications nowadays, performing human-centered tasks at reasonable cost [1]. They aim to achieve a high level of functionality, flexibility and efficiency at the same time, acting in complex, unstructured and potentially hazardous environment. In addition, the quality and safety of HumanRobot Interaction (HRI) is key to intelligent personal robots.

One of the cornerstones of recent advancement in HRI is the evolution of descriptive logics, formal languages, ontologies and community vocabularies, nurturing on an expanding user base.

*óbuda University, Budapest, Hungary - haidegger@ieee.org \& Austrian Center for Medical Innov. and Techn. (ACMIT), Wiener Neustadt, Austria

${ }^{\dagger}$ Distributed Systems Laboratory (LaSiD), UFBA, Brazil - marcoseb@dcc.ufba.br

¥Polytechnic Institute of Castelo Branco / Technical University of Lisbon, Center of Intelligent Systems, IDMEC / LAETA, Portugal paulo.goncalves@ipcb.pt

${ }^{\aleph}$ The American University in Cairo, Cairo, Egypt - maki@aucegypt.edu

${ }^{£}$ Monash University, Sunway campus, Selangor, Malaysia veera.ragavan@monash.edu

$\S$ Dept. of Electrical and Computer Engineering, University of New Brunswick, Canada - howard@unb.ca

IDept. of Electronics, Information and Bioengineering (DEIB), NearLab Medical Robotics, Milan, Italy - alberto.vaccarella, roberta.perrone\}@mail.polimi.it

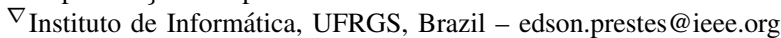

Ontologies, defined as the formal description of the concepts and relationships for an agent or a community of agents, are essential for HRI, especially for representing knowledge among humans and robots [2]. Ontology can be considered as an organized form of shared knowledge. Mathematically it can be defined as a tuple $\langle S, A>$, where $S$ is the signature set which can be broken down into three sets of concepts, relations and instances, while $A$ is a set of axioms specifying the domain vocabulary [2], [3].

When developers and researchers discuss and analyse service robotic concepts in their domain $D_{\mathrm{SR}}$, they use representation languages such as $L_{\mathrm{UML}}$ or $L_{\mathrm{OWL}}$ to analyse and communicate concept terminology and relations. OWL is supported by most semantic editing and reasoning software, such as Pellet and SWRL. OWL allows only two types of properties: object property and data type property. The former links objects with others, while the latter links objects to some defined values.

Ontologies can facilitate the development of robotic applications in various ways:

- Providing a consistent set of terminology (domain vocabulary), concepts, definitions, relations, domain axioms and taxonomy;

- Enabling procedural description of complex tasks, environment, etc;

- Providing a repository of knowledge that can be shared among various robots;

- Highlighting new relations through the analysis of data generated using ontologies.

\section{The Rise of Robotic Technology in the MEDICAL FIELD}

Robots have been used for surgical applications since the mid 1980s. Today, there are more than 3,500 surgical robots around the world performing hundreds of thousands of surgeries every year. Most commonly, master-slave systems (such as the da Vinci from Intuitive Surgical Inc.) help medical experts to overcome accuracy, time and space challenges. Through telepresence, physicians receive sensory feedback via the HRI, and may interact with patients remotely through teleoperation. These systems are used to execute tasks while being guided by humans, yet still under strict supervision. In the mean time, robots can perform sensor-driven tasks autonomously as well, supporting diagnosis and patient treatment [4].

Image-Guided Surgery (IGS) means the accurate correlation and mapping of the operative field to a pre-operative 
image or intra-operative (e.g., ultrasound [5], fluoroscopy) data set of the patient via sensory information (optical, mechanical or electromagnetic tracking). IGS provides better equipment positioning accuracy or guidance of mechatronic systems, and has primarily been used in neurosurgery, pediatrics, orthopaedics and Ear, Nose and Throat (ENT) surgery-domains that have been more exposed to novel technology [6].

Additional sensors may also be employed in the operative room, e.g., force sensors and Inertial Measurement Units (IMUs). Control actions can be taken once sensor information is processed. Forces and torques applied on the patients body are sensed and converted into haptic feedback.

\section{Ontologies for Medical Robots}

The development of ontologies for service robots (including medical) aims to produce a consensual and generic list of concepts and terminology for the robotics community at large.

A general framework of knowledge representation for robotic reasoning is defined in the scope of the IEEE Ontologies for Robotics and Automation Workgroup (ORA WG) [7]. ORA WG consists of 50 members from 20 different countries drawn from industry, academia and government organizations around the world. The draft of the complete IEEE RAS ontology is planned to be published in early 2014 [7]. The group undertakes tasks such as merging/aligning partial ontologies, evaluating distributed ontologies and extracting concepts from high-level process descriptions. The domain vocabulary currently under development will undergo reviews by domain experts and industry personals.

A notable effort is the REHABROBO-ONTO from Sabanci University, focusing on the development and maintenance of a public ontology for rehabilitation robotics [8]. It is enabled with an intelligent user-interface, called REHABROBO$Q U E R Y$. In this domain, the environment, i.e., context space is a key factor, since the ontologies for these kind of robots must be modular to be applicable to a large number of scenarios and application areas [9]. The application fields are identified by the IEEE RAS Technical Committee on Service Robots [10], in accordance with current standardization trends (at ISO and IEC)[11].

\section{CURRENT EFFORTS FOR SURGICAL ROBOT ONTOLOGIES}

To the authors' knowledge, there is no generic framework for process modeling and formal language description of robot-assisted surgery. Surgical tasks follow a strict clinical workflow, practiced for years by a surgeon. Although automating surgical applications and sub-tasks will require proper description of procedures and workflows, few projects focus on surgical ontologies. One example is the European Robotic Surgery (EuRoSurge) FP7 project (www.eurosurge.eu), lead by the Laboratory for Teleoperation and Autonomous Intelligent Robots (ALTAIR) at the University of Verona. EuRoSurge fosters component-based software architecture for computer- and robot-aided surgery research and manufacturing, based on different level of ontological modeling. It includes the creation of a glossary and ontology for cognitive surgical robotics.

Besides EuroSurge, there are more specific initiatives. Two surgical domains have long been in the forefront of sensorand computer-technology integration, mostly due to their need of high accuracy:

1) Neurosurgery has always been the leading application area of cutting edge medical technology. The NeuroMate robot (formerly developed by Innovative Medical Machines Inc., Lyon, France, then owned by ISS and lately by Schaerer Mayfield NeuroMate AG, Lyon, France) was the first neurosurgical robotic device to obtain the CE certificate in Europe, and then the US Food and Drug Administration's (FDA) approval in 1997 for stereotactic neurosurgical procedures [12].

2) In orthopaedics, navigation technology, templates and image-guided tools have been employed from the first half of the $20^{\text {th }}$ century. Beyond, the ROBODOC, designed for knee surgery (formerly developed by Integrated Surgical System Inc. in Sacramento, CA, currently owned by the Korean Curexo Inc.) was the first robot to perform automated procedures on humans in 1992 [13].

Below, we present two examples of surgical ontologies containing robots applied in the above mentioned fields.

\section{A. Neurosurgery Robotic Ontology}

The Neurosurgery Robotic Ontology (NRO)—being developed at the Politechnico di Milano-includes concepts for the pre-operative and intra-operative phases of neurosurgery [14]. It was founded on the identification of major concepts, relations and cardinality in the work domain, based on information in Rhoton's work [15]. Further knowledge was obtained through interviews with two neurosurgeons, Dr. F. Cardinale (Claudio Munari Epilepsy Surgery Centre, Niguarda Hospital, Milan) and Prof. L. Bello (Humanitas Hospital, Milan).

With these concepts, it was possible to define a surgical workflow and components. To build the ontology, three steps were followed:

1) Definition of domain's concepts (classes) by textbooks and interviews with surgeons;

2) Hierarchical organization of concepts, concept attributes, restrictions and relations among concepts (properties);

3) Definition of instances of concepts and population of the ontology.

Once the foundation of the ontology has been developed, it is necessary to decide which concepts need to be included in the ontology. Then ambiguities need to be resolved, since different words from different data sources may refer to the same concept. It is important to note that surgeons with different background and nationality may have different interpretations.

In addition, the Internet can be used to connect related concepts not yet connected, in order to facilitate gathering 


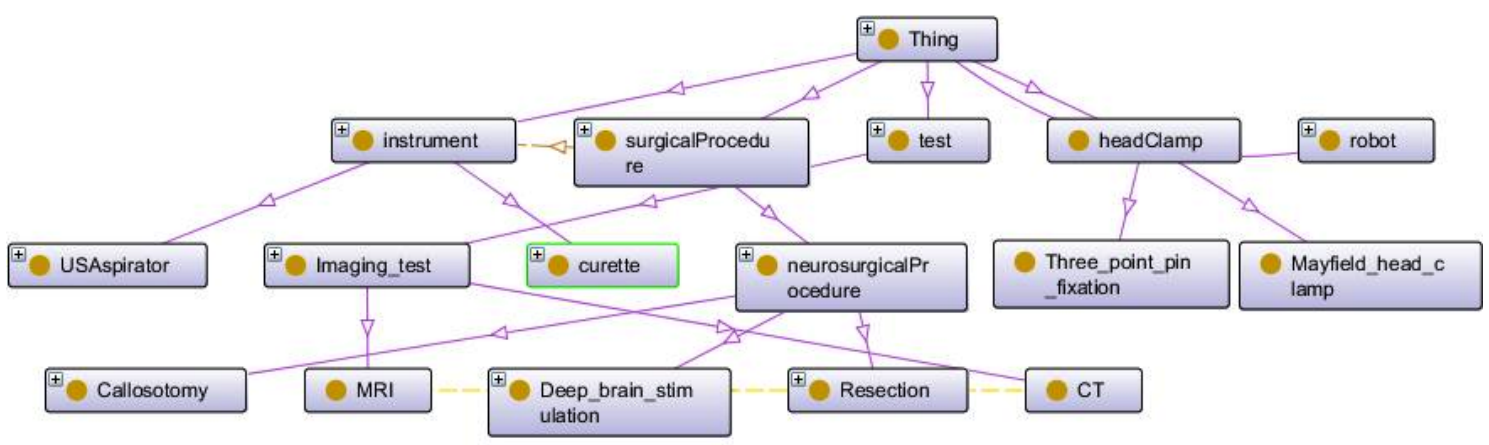

Fig. 1. An example ontology derived from the neurosurgical domain. Solid lines represent taxonomic relationships between concepts, while dotted lines represent object properties between classes.

and sharing data, information and knowledge within the community. This can be implemented using Resource Description Framework (RDF) and Uniform Resource Identifier (URI).

For the developed ontology for neurosurgery, every concept is linked to DBpedia ontology's resources and UMLS ontology [16]. DBpedia (aksw.org/Projects/DBpedia.html) is a community effort to extract structured information from Wikipedia and to make this information available on the Internet (dbpedia.org). It allows users to make sophisticated queries to Wikipedia and to link other datasets on the Internet to Wikipedia. Concepts are connected to DBpedia with the built-in property owl:sameAs which connect an individual to another individual. A statement that makes use of this property asserts that two URI references actually refer to the same thing. In particular, it should be used $A$ owl:sameAs $B$, if anything that can be said for $A$ is true for $B$ and vice versa. Thus, the two ontologies are linked and a large amount of information can be exploited through DBpedia (e.g., synonyms, translations, documents and properties).

There are many popular graphical languages to model ontologies (more or less compliant to human cognition). However, a model becomes an ontology only if it is both machine-readable and agreed-upon. For building and sharing this ontology, Protègè (3.4.6:2011) was used. Currently, the most populated class is the instruments class. It has a long list of tools used in the two hospitals. For each instrument, dimensions and weight are stored. Fig. 1 shows a diagram of the main classes of the NRO built with the information collected so far.

At this stage, the neurosurgery ontology contains:

- 452 classes, e.g., instrument, patient, robot, surgical procedure, personnel and actions to be performed during a surgical procedure;

- 42 properties, 30 of which are object properties and 12 of which are datatype properties;

- 180 individuals, most of them are instrument, patient and symptom classes;

The NRO can also be used help in building a classifier for differentiating types of epilepsy that could affect a patient and that manifest with a set of visible symptoms. The preoperative phase is characterized by concepts that describe the pathology afflicting the patient: in particular it is taken into account that the epilepsy disease most of the time can be treated using surgical procedures. (E.g., EEG data and semiology of epilepsy patients is described.) Due to the fact that the epileptogenic focus can characterize epilepsy seizure, the neurosurgery ontology imports the existing ontology available at (www.bioportal.com) in order to map the brain with Brannan areas, in such a way that every epileptogenic focus maps to a specific area of the brain.

Ontologies can be combined with classification algorithms. For example, patient classification can be realized using data from real patients and attributes, such as ictal signs. The ontological modeling, the hierarchical and graph structure can help to determine the correlation between ictal symptoms observed and/or not observed during a seizure.

In the future, the ontology will be extended for the classification of robots that are commonly used in neurosurgical treatment for epilepsy [17], [18], [19], among others. In order to extend the ontology, it is necessary to determine what attributes can describe individuals in the domain and the attributes' type (e.g., number of operations every year is an integer).

Our ontology provides a specification of the neurosurgical domain and helps surgeons to reason using available data. In the future, the extended ontology can also be used directly to support robotic treatment delivery, based on real-time sensory information acquisition.

\section{B. Ontology for orthopaedics}

The application presented in this section is related to Hip Resurfacing (HR) prosthesis surgery. It is an alternative to Total Hip Replacement (THR), especially for younger patients that may require hip joint revisions in the future [20]. Hip resurfacing can be implemented using several approaches, however, the Birmingham Hip resurfacing device (Smith \& Nephew Inc.) is the most widely used because of its accuracy. On the downside, HR is more complex than THR, and requires precise and accurate following of the prescribed surgical plan and workflow, therefore various sensor and navigation systems have already been developed to support these operations.

Ontologies in this case are used to model the workflow/knowledge required to accomplish each surgery 


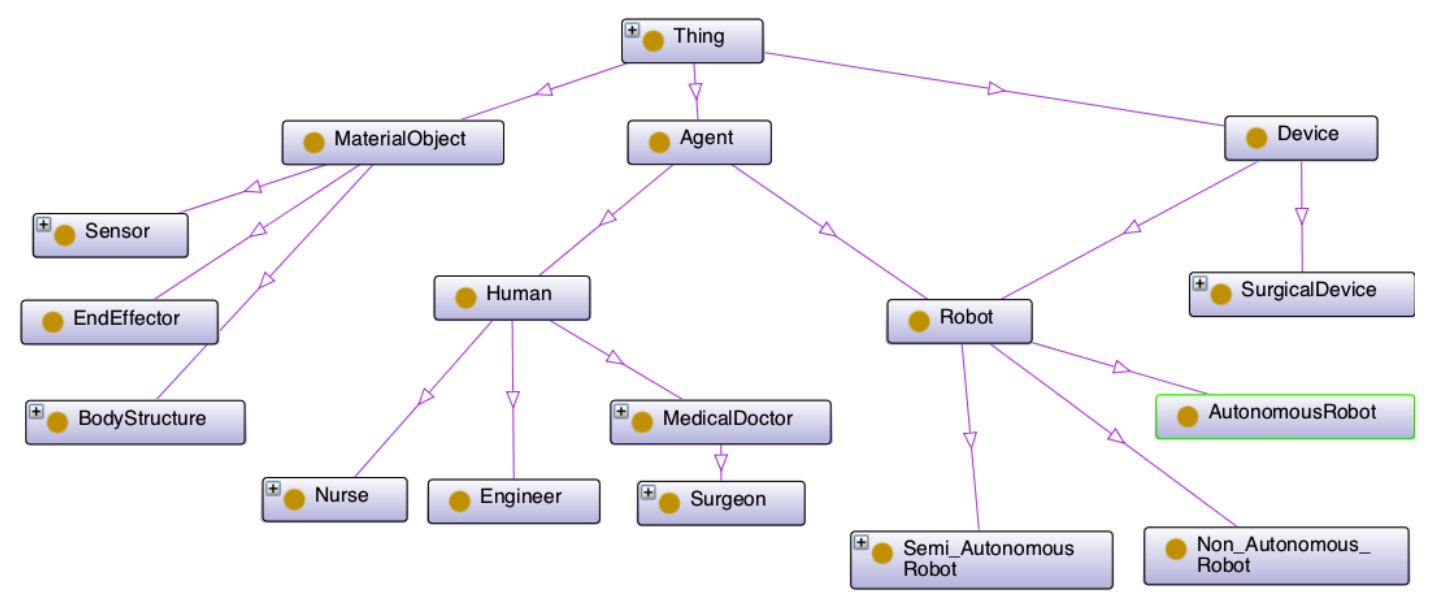

Fig. 2. A fraction of the orthopedic ontology, related to the components of the System, obtained from Protégé. Solid lines indicate class hierarchy.

task in details, allowing successful surgeries, and also to represent that knowledge in a machine-readable format. The ontological model obtained, i.e., its formal description, is of major interest to setup a service robot, e.g., a robot manipulator, that will perform the surgery in a co-worker scenario with the surgeon, delivering the expected increased accuracy and precision. This work is developed under the HIPROB (http://www.echord.info/wikis/website/hiprob) [5] and ECHORD projects (http://www.echord.info/wikis/website/home).

The ontology project was initiated when the $I D$ MEC/Polytechnic Institute of Castelo Branco, Portugal was asked to automate HR surgery, following contacts with surgeons from Lisbon and Castelo Branco main Hospitals. A set of interviews was conducted with the surgeons to better define the domain and scope of the approach to establish HR Robotic Surgery (HRRS). For creating the ontology within these projects, a process is developed, called ontology development 101 [21]. This includes:

1) Reviewing existing ontologies;

2) Identifying classes and properties;

3) Identifying and implements an upper ontology;

4) Implementing the ontology in a formal representation.

Existing ontologies from the medical and the robotics fields were reviewed, such as $N C B O$ BioPortal [22], the Open Biological and Biomedical Ontologies [23], OpenCyc [24], the SOCAS Ontology [25], the Open Robots Ontology (ORO) [26] and KnowRob [27]. They are considered to be the main sources of contents and cover almost all the knowledge needed to develop the ontology for orthopedic robotic surgery.

The classes and their properties were identified using the existing definitions on the base ontologies, like BodyStructure, Surgeon from the BioOntology [22] and SurgicalDevice adapted from SOCAS [25]. From this and the functional requirements obtained from the surgical workflow for HR surgery, new classes and properties are defined for the pre- and intra-operative surgery phases, e.g., IntraOperativePhase and PreOperativePhase. Also Device and Agent were defined-along their sub-classes-following the work of Prestes et al. [3]). Fig. 2 presents the main components for the Orthopaedic Ontology.

It is possible to merge or extend the ontology, e.g., a particular property from the orthopaedic ontology, applied to the HR surgery using ultrasound navigation [5], is that the ultrasound probe US_probe (a sub-class of US_imaging) isAttachedTo the EndEffector and IsUsedIn the IntraOperativePhase. Computer tomography imaging $C T$ _imaging) isUsedIn the PreOperativePhase. Fig. 3 illustrates the main relations within the HR surgery process, where it highlights the Human-Robot Cooperation, i.e., the Surgeon can Hold the Bone_Drill, that IsAttachedTo the Semi-Autonomous_Robot. The robot allows a precise drilling, by constraining surgeons' involuntary movements. In this work, the femur IsRepresentedBy a PointCloud that enables the registration from the intra and pre-operative obtained bone models [5].

The next step is to implement an upper ontology in the HIPROB Software Tool [28]. For that, OpenCyc is chosen, because it is interchangeable amongst the previous presented robotic and biomedical ontologies, and also provides a basic structure, generalizable to domain specific ontologies. OpenCyc is used as the main source to design the structure and also to define HRRS ontology classes and properties.

The ontology is implemented in OWL, and a set of classes are defined specifically for the HRRS setup, e.g., Bone Drill, US_Probe. The knowledge management module for cognitive architectures in robotics $O R O$, is used to map the robot services to the orthopedic ontology [26]. ORO is an open-source framework that can be used within the ROS framework to control a robot. This model includes, amongst other features, the capability of knowledge classification based on SWRL rules, using Pellet-an OWL 2 Reasoner for Java (http://clarkparsia.com/pellet).

Surgical tools, some to be used by the robot, e.g., Bone Drill, and others to be used by the surgeons are defined, i.e., common surgical tools like scissors, or orthopedic surgery specific ones, like reamers or alignment jigs. For the HRRS, knowledge from the human body must also 
be shared with the robot via the ontology, e.g., the femoral head and the acetabulum. HRRS employs other equipment, e.g., a $K U K A-L W R$ torque-controlled manipulator and optical tracking systems, e.g., an NDI Polaris Spectra. These are also included in the ontology.

For HRRS, multiple sensors and sensory systems are needed (e.g., medical imaging systems) to obtain 3D information of the femur. In an orthopaedic ontology, various imaging sources can be incorporated, such as Magnetic Resonance (MR), Computed Tomography (CT), X-ray and ultrasound. In the current application, the sensors used to collect data about the surgical environment are depicted in Fig. 4, ultrasound is used for intra-operative imaging and $\mathrm{CT} / \mathrm{MR}$ for the pre-operative stage. To obtain a precise $3 \mathrm{D}$ position of the femur during the intra-operative procedure, an Optical_Tracker is also used to obtain the bone's PointCloud.

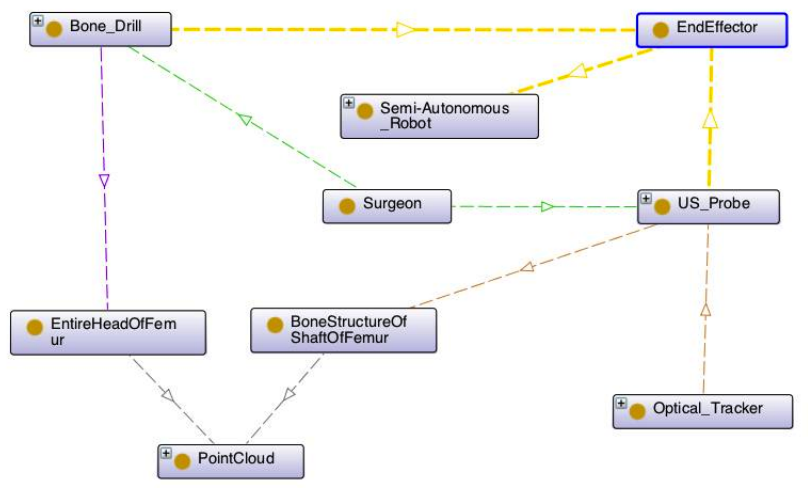

Fig. 3. A fraction of the orthopaedic ontology, related to the HRRS process, obtained from Protégé. Dashed lines indicate connections between classes.

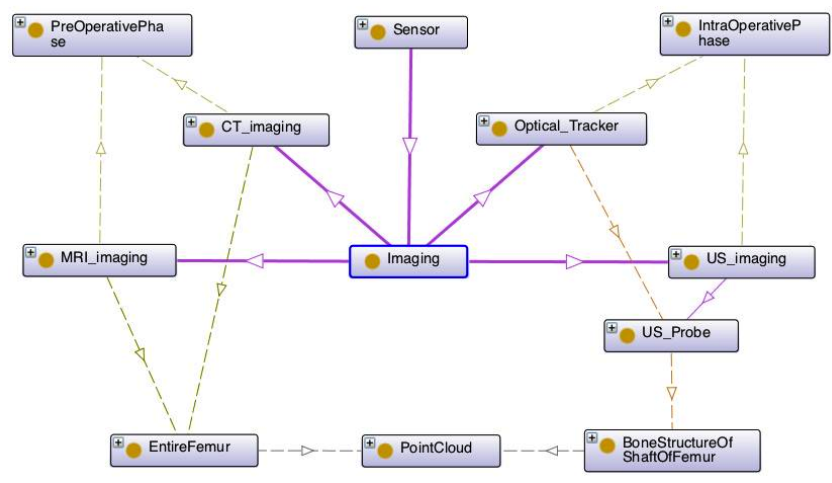

Fig. 4. A fraction of the orthopedic ontology, related to the HRRS sensors, obtained from Protégé. Solid lines indicate class hierarchy and dashed lines indicate connections between classes.

\section{The Future Role of Cognitive Technologies in MEDiCAL Robotics}

Compared to other domains, there are few service robots developed for medical applications. The major reason is the high performance requirements and constrained legal environment for these systems. For information accumulation, reasoning and decision making, autonomous or semiautonomous systems currently does not have the internal capabilities to deal with newly arising complex surgical situations.

Ontologies can help to build entire platforms for new robotic services. Recently, cloud computing has been applied to robotics in order to provide "Robots as a Service" (RaaS), which can be dynamically combined to give support to the execution of specific applications. There is a handful of initiatives towards medical RaaS, and a few successful pilot projects has been demonstrated globally.

Shared knowledge is essential, e.g., for medical treatment planning, where large, semantically labeled, prior procedures can be used to derive an optimal treatment delivery plan onthe-fly, along with a projection of the possible outcome. Systematic description of the operating room workflow would make surgical robots compatible, and tasks more easy to share. Outsourcing heavy computational tasks in sensing and robot control could be advantageous in the future, while larger and more detailed patient images and recordings can also be created this way. IBM's famous supercomputer, Waston is reported to apply its computational capacity and reasoning capability to the medical domain. It has processed large patient data for early diagnosis and potential health risk identification [29].

One of the key aspects of HRI is the human end-user's emotional reaction, when working with a robot, or getting into physical interaction. Human-robot communication and safety need to be formerly tackled by the community, which could be facilitated by ontologies, and standards built on the top of these [2]. Design and software driven product development are two important aspects of ontology development. A successful design of future service robots will be based on detailed knowledge of applicable technologies and methodologies.

\section{Future WORK}

The ontologies outlined in this paper represent only a small subset of the many emerging knowledge domains that will eventually help tackling complex medical problems. However, these ontologies must be organized in hierarchy, merged and synchronized [3]. It is also essential that the clinical community accepts these ontologies, and validate it through everyday use in the operating rooms. Our group therefore is working on the extension of the presented ontologies and their integration to the IEEE RAS Core Ontology. This work is supposed to be finished in 2015, and being validated by a selected group of experts first, and then, by the larger community. The aim of the authors is to develop clear, precise and easy-to-use ontologies for robot designers as well as robot end-users, yet only the first steps have been taken.

The authors are also working together with the International Organization for Standardization and International Electrotechnical Commission (ISO/IEC) TC 184 (Technical Committee on Automation Systems and Integration), SC 2 (Sub-Committee on Robots and Robotic Devices), JWG 9 (Joint Work Group on Standard for Medical Robots) to nest these ontologies into the future standards for medical robots. 


\section{CONCLUSION}

Success of all service robot applications rely on humans using those services, or benefiting from the results. This is especially true in medicine, therefore human-robot interaction, robotic perception, robotic knowledge, robotic intelligence and safety - amongst others - are critical issues that robotic researchers need to address. Robot-assisted surgery is an emerging domain. A robot may augment or replace the human operator, by manipulating endoscopic tools, performing complex procedures or using smart tools. It is understood that further development of autonomous functions and deployment of more capable robots need standardized ontologies that can accumulate and generate knowledge automatically.

In this paper, ontologies for medical robots were described. Two examples, developed by the authors, were demonstrated for neurosurgical orthopaedics applications, describing their basic structure and capabilities. The use of ontologies could facilitate advanced service robot applications in the domain of health care, and bringing us closer to structurally analyze and understand complex procedures, such a surgeries.

\section{ACKNOWLEDGMENT}

The IEEE-SC WG is supported by the IEEE Robotics and Automation Society. This work is partially supported by the Strategic Project, PEst-OE/EME/LA0022/2011, through FCT (under IDMEC-IST, research group: IDMEC/LAETA/CSI), FCT project PTDC/EME-CRO/099333/2008 and EU-FP7ICT-231143 project ECHORD. The authors acknowledge the EuRoSurge FP7-ICT-2011-7-288233 project, the support of Brazilian CNPq. T. Haidegger is a Bolyai Fellow.

\section{REFERENCES}

[1] Habib, M. K., "Service robots and humanitarian demining," Mobile Robotics: Toward New Applications, pp. 449-480, 2012.

[2] T. Haidegger, M. Barreto, P. Gonçalves, M. K., V. Ragavan, and $\mathrm{H}$. Li, "Applied ontologies and standards for service robots," Robotics and Autonomous Systems, pp. 1-19, 2013, in press, DOI: 10.1016/j.robot.2013.05.008.

[3] E. Prestes, J. Luis Carbonera, S. Rama Fiorini, V. A. M. Jorge, M. Abel, R. Madhavan, A. Locoro, P. Gonçalves, M. E. Barreto, M. Habib, A. Chibani, S. Gérard, Y. Amirat, and C. Schlenoff, "Towards a core ontology for robotics and automation," Robotics and Autonomous Systems, vol. in press, 2013.

[4] R. Taylor and P. Kazanzides, "Medical Robotics and ComputerIntegrated Interventional Medicine," Advances in Computers: Emerging Technologies, vol. 73, pp. 219-263, 2008.

[5] P. M. B. Torres, J. Sanches, P. J. S. Goncalves, and J. M. M. Martins, "3D femur reconstruction using a robotized ultrasound probe," in 4th IEEE RAS \& EMBS Intl. Conf. on Biomedical Robotics and Biomechatronics (BioRob), 2012, pp. 884-888.

[6] G. Fichtinger, P. Kazanzides, G. D. Hager, A. M. Okamura, L. L. Whitcomb, and R. H. Taylor, "Surgical and Interventional Robotics: part II," IEEE Robotics and Automation Magazine (RAM), vol. 15, no. 3, pp. 94-102, 2008.

[7] C. Schlenoff, E. Prestes, R. Madhavan, P. Gonçalves, H. Li, S. Balakirsky, T. Kramer, and E. Miguelanez, "An IEEE standard ontology for robotics and automation," in Proc of the IEEE/RSJ Intl. Conf. on Intelligent Robots and Systems (IROS), 2012, pp. 1337-1342.

[8] Z. Dogmus, G. Gezici, V. Patoglu, and E. Erdem, "Developing and maintaining an ontology for rehabilitation robotics," in Proc. of the $4^{\text {th }}$ Intl. Conf. on Knowledge Engineering and Ontology Development (KEOD), 2012, pp. 389-395.
[9] J. Hallam and H. Bruyninckx, in European Robotics Symposium 2006, ser. Springer Tracts in Advanced Robotics, H. I. Christensen, Ed. Springer Berlin Heidelberg, 2006, vol. 22, pp. 1-14.

[10] "IEEE RAS technical committee on service robots," 2012, http://www. service-robots.org/

[11] G. S. Virk and T. Haidegger, "Classification guidelines for personal care robots-medical and non-medical applications," in Proc. of the IEEE IROS Workshop on Safety in Human-Robot Coexistence \& Interaction, 2012, pp. 33-36.

[12] T. Haidegger, L. Kovacs, G. Fordos, Z. Benyo, and P. Kazanzides, "Future trends in robotic neurosurgery," in Proc. of the $14^{\text {th }}$ NordicBaltic Conf. on Biomedical Engineering and Medical Physics (NBC), 2008, pp. 229-233.

[13] P. Kazanzides, "Robot assisted surgery: the ROBODOC experience," in Proc. of the 30th Intl. Symp. on Robotics (ISR), Tokyo, 1999, pp. 281-286.

[14] R. Perrone, "Ontological modeling for Neurosurgery: application to automatic classification of temporal and extratemporal lobe epilepsies," MSc thesis, Politechnico di Milano, 2012.

[15] A. L. Rhoton, "Operative techniques and instrumentation for neurosurgery," Neurosurgery, vol. 53, no. 4, pp. 907-937, 2003.

[16] D. M. Pisanelli, A. Gangemi, and G. Steve, "An ontological analysis of the umls metathesaurus," in Proc. of AMIA Symp., vol. 5, 1998, pp. $810-814$.

[17] Eljamel, M., "Robotic application in epilepsy surgery," Intl. J. of Medical Robotics and Comp. Assisted Surgery, vol. 2, pp. 233-237, 2006.

[18] Varma, T. and Eldridge, P., "Use of the NeuroMate stereotactic robot in a frameless mode for functional neurosurgery," Intl. J. of Medical Robotics and Computer Assisted Surgery, vol. 2, pp. 107-113, 2006.

[19] W. J. Spire, B. C. Jobst, V. M. Thadani, P. D. Williamson, T. M Darcey, and D. W. Roberts, "Robotic image-guided depth electrode implantation in the evaluation of medically intractable epilepsy," Neurosurgery Focus, vol. 25, no. 3, 2008.

[20] W. Bargar, A. Bauer, and M. Börner, "Primary and revision total hip replacement using the robodoc system," Clinical Orthopedics and Related Research, vol. 354, pp. 82-91, 1998.

[21] F. N. Natalya, L. Deborah, and McGuinness, "Ontology development 101: A guide to creating your first ontology," Stanford Knowledge Systems Laboratory Technical Report KSL-01-05 and Stanford Medical Informatics Technical Report SMI-2001-0880, 2001.

[22] N. F. Noy, N. H. Shah, P. L. Whetzel, B. Dai, and al., "Bioportal: Ontologies and integrated data resources at the click of a mouse," Nucleic Acids Research, vol. 37, no. s2, pp. 170-173, 2009.

[23] B. Smith, M. Ashburner, C. Rosse, J. Bard, W. Bug, W. Ceusters, and al., "The OBO Foundry: Coordinated evolution of ontologies to support biomedical data integration," National Biotechnology, vol. 37, pp. 1251-1255, 2007.

[24] "OPENcyc," 2012, http://www.cyc.com/cyc/opencyc/overview

[25] D. Neumuth, F. Loebe, H. Herre, and T. Neumuth, "Modeling surgical processes: A four-level translational approach," Artificial Intelligence in Medicine, vol. 51, pp. 147-160, 2011.

[26] S. Lemaignan, R. Ros, L. Mösenlechner, R. Alami, and M. Beetz, "ORO, a knowledge management module for cognitive architectures in robotics," in Proc. of the IEEE/RSJ Intl. Conf. on Intelligent Robots and Systems (IROS), 2010, pp. 3548-3553.

[27] M. Tenorth and M. Beetz, "Knowrob—-knowledge processing for autonomous personal robots," in Proc. of the IEEE/RSJ Intl. Conf. on Intelligent Robots and Systems (IROS), 2009, pp. 4261-4266.

[28] P. J. S. Gonçalves, P. M. B. Torres, F. Santos, R. António, N. Catarino, and J. M. M. Martins, "On the development and simulation of a robotic ultrasound guided system for orthopedic surgical procedures," in Proc. of the 13th IEEE Intl. Conf. on Mobile Robots and Competitions, 2013, pp. 38-43.

[29] C. Johnathan, "The robot will see you now," The Atlantic, March, 2013, www.theatlantic.com/magazine/archive/2013/03/ the-robot-will-see-you-now/309216/ 